\title{
PEG-liposomal oxaliplatin potentialization of antitumor efficiency in a nude mouse tumor-xenograft model of colorectal carcinoma
}

\author{
CHUANG YANG ${ }^{1,2}$, HAI-ZHONG LIU ${ }^{3}$, WEI-DONG LU ${ }^{1}$ and ZHONG-XUE FU ${ }^{1}$ \\ ${ }^{1}$ Department of Gastrointestinal Surgery, First Affiliated Hospital, Chongqing Medical University, \\ Chongqing 400016, Chongqing; ${ }^{2}$ Department of Hepatobiliary Surgery, Mianyang Third People of \\ Hospital, Mianyang 621000, Sichuan Province; ${ }^{3}$ Department of Gynecology \& Obstetrics, Second \\ Affiliated Hospital, Chongqing Medical University, Chongqing 400016, Chongqing, P.R. China
}

Received January 14, 2011; Accepted February 23, 2011

DOI: $10.3892 /$ or.2011.1238

\begin{abstract}
The non-selectivity of chemotherapeutics between normal tissue and pathological sites poses a challenge for the treatment strategy for advanced colorectal carcinoma. To obtain sufficient antitumor activity, optimization of the therapeutic regimen is of great importance. We investigated PEG-liposomal oxaliplatin potentialization of antitumor efficiency in a nude mouse tumor-xenograft model of colorectal carcinoma. A tumor-bearing nude mouse model, intravenous injections of (Dio)-labeled PEG-liposomes via tail vein and fluorescence imaging with in vivo imaging system were employed. Mice were treated with free L-oHP, PEG-liposomal L-oHP via the tail vein, followed by analysis of the accumulation of L-oHP in tumor tissues by high-performance liquid chromatography (HPLC), observation of the tumor volume and the survival rate. Apoptosis and proliferation of tumors were detected by TUNEL assay and immunohistochemistry. The mRNA and protein levels of Bcl-2, Bax, caspase-3 (P17) and Ki-67 were determined by RT-PCR and Western blotting. Fluorescence imaging with in vivo imaging showed PEG-liposome targeting in tumor tissues. After intravenous injections of PEG-liposomal oxaliplatin, tumor tissue maximum accumulation of L-oHP was $9.37 \pm 0.79 \mu \mathrm{g} / \mathrm{g}$ at $24 \mathrm{~h}$; The tumor volume was significantly suppressed, and mice showed longer survival, compared with the free oxaliplatin group. Apoptosis increased, but proliferation decreased in tumor tissues. The mRNA expression of Bcl-2 and Ki-67 was down-regulated, while Bax and caspase-3 expression was up-regulated. Protein expression of Bcl-2 was down-regulated, while Bax and P17 expression was up-regulated. The results indicate that PEG-liposomal oxaliplatin can improve antitumor efficiency in a nude mouse tumor-xenograft model of colorectal carcinoma.
\end{abstract}

Correspondence to: Dr Zhong-Xue Fu, Department of Gastrointestinal Surgery, First Affiliated Hospital, Chongqing Medical University, Chongqing 400016, Chongqing, P.R. China E-mail: fzx990521@sina.com

Key words: apoptosis, colorectal carcinoma, liposomes, oxaliplatin

\section{Introduction}

Colorectal carcinoma (CRC) is one of the most common cancers that affects men and women equally. For advanced stage CRC, chemotherapy still remains the main therapeutic strategy (1).

Oxaliplatin (L-oHP), is a third generation of platinum antitumor compound. It has been approved for standard first- and second-line treatment of metastatic or advanced stage colorectal cancer in combination with the infusion of 5-fluorouracil (5-FU)/leucovorin (FOLFOX) $(2,3)$. However, its clinical efficiency is limited by the dose-limiting side-effects. The cytotoxic drugs have apparent toxicity on human body such as neurotoxicity, gastrointestinal reaction and cardiotoxicity. (4), moreover, the non-selectivity of cytotoxic drugs between normal tissue and pathological site pose a challenge for the treatment strategy for tumors. Conventional chemotherapy is not as effective in colorectal cancer as it is in other cancers, because the drug does not reach the target site in effective concentrations $(5,6)$. Thus, effective treatment demands increased dose size, which may lead to side-effect.

To overcome these limitations, new delivery systems with alternative drug release mechanism have been suggested. Targeting of drugs specifically to the colon is advantageous for the treatment. Liposomes were one of the first nanomolecular drug delivery systems to show increased delivery of smallmolecular weight anticancer drugs to solid tumors by altering the biodistribution of associated drugs $(7,8)$. Liposomes have repeatedly shown to be able to improve the therapeutic index of a variety of drugs. Moreover, 1,2-distearoyl-snglycero-3-phosphoethanolamine- $\mathrm{N}$-(maleimide(polyethylene glycol)-2000) (DSPE-PEG2000) modification to the surface of the liposome may preventing interactions with the biological in vivo environment, then extend the circulation lifetime of the liposome (9-11). This results in extensive extravasation of the liposomes due to the tumor selective enhanced permeability and retention effect, ultimately leading to enhanced accumulation of the liposomes in the tumor interstitium (12). According to these theories, the liposome-containing drugs will show markedly enhanced antitumor activity, dual targeting approach to both endothelial cells in angiogenic vessels and tumor cells. 
Our prophase experiment showed that the PEG-liposomes enhanced antitumor activity for colorectal carcinoma cell lines (13). In this study, we investigated the therapeutic activity of PEG-liposomal L-oHP in a nude mouse solid tumor model.

\section{Materials and methods}

Reagent. PEG-liposomal L-oHP, Dio-labeled liposomes (previous preparation). Rabbit polyclonal antibodys of $\mathrm{Bcl}-2$, Bax were purchased from Santa Cruz Biotechnology Inc., rabbit polyclonal anti- $\beta$-actin and goat anti-rabbit $\mathrm{IgG}$, peroxidase conjugate secondary antibody were obtained from Bioscience company of America. Activated-caspase-3 (P17) was obtained from Bioworld Company, Ki-67 was from Abcam Inc., TUNEL Kit was purchased from Rochs Gemer Inc.

Animals and tumor cell line. Human colorectal carcinoma cell line (SW480) was obtained from Institute of Life Science of Chongqing Medical University, and maintained in RPMI1640 (Sigma, St. Louis, MO) medium containing 10\% fetal bovine serum (FBS) (HyClone, Logan, UT) with streptomycin/penicillin in an incubator under $5 \% \mathrm{CO}_{2}$ at $37^{\circ} \mathrm{C}$. Female BALB/c nude mice, 3 weeks old, weighing 18-20 g, were obtained from center of laboratory animals Chongqing Medical University. All animal experiments were evaluated and approved by the Animal and Ethics Review Committee (certificate number: SCXK(jing)2009-0004).

Targeting of Dio-labeled liposomes in mouse tumor-xenograft. Fale BALB/c nude mice were inoculated subcutaneously at the inguen region with $\mathrm{SW} 480$ cells $\left(2 \times 10^{7} /\right.$ mouse $)$ in a volume of $200 \mu \mathrm{l}$ (PBS). On days 15 after tumor inoculation, the tumor volume reached approximately $100 \mathrm{~mm}^{3}$. Then intravenous injections of Dio-labeled liposomes $(0.5 \mathrm{nmol} / \mathrm{g})$ was via tail vein. At $24 \mathrm{~h}$, post-injection, nude mice were maintained anesthetized with isoflurane, and fluorescence imaging with In vivo Imaging system (Mastro Ex, USA) with affiliated anesthesia device. The tumors were dissected, and then frozen-sections $(10 \mu \mathrm{m})$ were prepared. Immunofluorescence staining for Hoechst ${ }_{33342}$ was performed to visualize nucleolus. Localization of PEG-lipsomes was observed in the tumor.

Accumulation of L-oHP in tumor tissue. On day 12 after tumor inoculation, mice were injected intravenously with either L-oHP solution ( $5 \mu \mathrm{g} / \mathrm{g}$ ) or PEG-liposomal L-oHP (5 $\mu \mathrm{g} / \mathrm{g} \mathrm{L}$-oHP). At 2, 12, 24, 48, $72 \mathrm{~h}$ post-injection, tumors were excised, and immediately washed twice with normal saline $(0.9 \% \mathrm{NaCl})$, wiped with filter paper, weighed $500 \mathrm{mg}$, and homogenized with $500 \mu \mathrm{l}$ of lysis buffer. The sample was centrifuged for $12,000 \mathrm{rpm}$ at $10 \mathrm{~min}$. The supernatant was collected, then added methyl cyanides $(1: 1 \mathrm{v} / \mathrm{v})$, vortexed and centrifuged $(10,000 \mathrm{rpm}, 10 \mathrm{~min}), 20 \mu \mathrm{l}$ of supernatant was injected into the high-performance liquid chromatography (HPLC) system for analysis of the content of L-oHP in the sample.

Therapeutic efficacy of PEG-liposomal L-oHP in the mouse tumor-xenograft. After successful inoculated subcutaneously transplantation tumor, the nude mice were random divided into three groups. Control $(n=6)$ : received intravenous injections of
5\% dextrose solution; free L-oHP $(\mathrm{n}=6)$ : received intravenous injections of $5 \mu \mathrm{g} \mathrm{L-oHP/g;} \mathrm{PEG-liposomal} \mathrm{L-oHP}(\mathrm{n}=6)$ : received intravenous injections of $0.5 \mathrm{nmol} / \mathrm{g}$ PEG-liposomal L-oHP (5 $\mu \mathrm{g} \mathrm{L-oHP/g).} \mathrm{Treatment} \mathrm{once} \mathrm{every} \mathrm{four} \mathrm{days,} \mathrm{the}$ antitumor activity was evaluated in terms of both tumor volume and the survival rate. The nude mice were sacrificed by deep anesthesia, the tumors were placed in liquid nitrogen immediately, for further experiments.

Tagged deoxynucleotide transferase (TdT) labelling. Tissue specimens were fixed in $4 \%$ paraformaldehyde and, when necessary, decalcified in $10 \%$ ethylene diamine tetraacetic acid (EDTA). The samples were dehydrated, embedded in paraffin, and sectioned into $5-\mu \mathrm{m}$ sections. To assess the presence of apoptosis, a standard TUNEL method was used. In paraffin sections, fragmented nuclear DNA associated with apoptosis was labeled in situ with digoxigenin-deoxyuridine (dUTP), and was introduced by terminal deoxynucleotidyltransferase (TdT), according to a standard method using Apop Tag Plus peroxidase kit (Roche). In order to avoid an overestimation of the TUNEL-positive cells, only cells that exhibited both morphologic features of apoptosis on light microscopy, but not associated with inflammation, and positive TUNEL staining for fragmented DNA were considered as positive. For negative control purposes, some slides were incubated with a labeling solution that did not contain Tdt.

Immunocytochemical staining. The slides were mounted as described above. Following treatment, the slides were exposed to a Ki-67 (1:100) antibody and incubated overnight at $4^{\circ} \mathrm{C}$ and subsequently exposed to horseradish peroxidase (HRP)labeled secondary antibody for $1 \mathrm{~h}$ at $37^{\circ} \mathrm{C}$, then colored using DAB. The proportion of positive cells (as determined by brown cytoplasm and nucleus) was counted. As a negative control, phosphate-buffered saline (PBS) rather than the primary antibody, was used.

Reverse transcription-polymerase chain reaction (RT-PCR). Total RNA was extracted using TRIzol (Takara, Dalian, China), then reverse transcripted. PCR amplification to detect differences among the samples was set as followed: 4 min at $94^{\circ} \mathrm{C}$ for initial denaturation; 30 cycles x $30 \mathrm{sec}$ at $94^{\circ} \mathrm{C}, 30 \mathrm{sec}$ at $59^{\circ} \mathrm{C}$, and $30 \mathrm{sec}$ at $72^{\circ} \mathrm{C}$ for Bcl-2, Bax; 30 cycles x $30 \mathrm{sec}$ at $94^{\circ} \mathrm{C}, 30 \mathrm{sec}$ at $56^{\circ} \mathrm{C}$, and $30 \mathrm{sec}$ at $72^{\circ} \mathrm{C}$ for Caspase-3; 30 cycles $x 30 \mathrm{sec}$ at $94^{\circ} \mathrm{C}, 30 \mathrm{sec}$ at $60^{\circ} \mathrm{C}$, and $30 \mathrm{sec}$ at $72^{\circ} \mathrm{C}$ for GAPDH (526 bp), Ki-67; $30 \mathrm{sec}$ at $54^{\circ} \mathrm{C}$, and $30 \mathrm{sec}$ at $72^{\circ} \mathrm{C}$ for GAPDH (230 bp). The following primer pairs were used: Bax (153 bp): 5'-GAT CGA GCA GGG CGA ATG GG-3' (forward primer); 5'-CAC GGC GGC AAT CAT CCT CT-3' (reverse primer). Bcl-2 (350 bp): 5'-CAG ATG GCA AAT GAC CAG CAGA-3' (forward primer), 5'-TGG CAG GAT AGC AGC ACA GGAT-3' (reverse primer). Caspase-3 (500 bp): 5'-GTG GAA TTG ATG CGT GAT G-3' (forward primer); 5'-GGA ATC TGT TTC TTT GCA TG-3' (reverse primer). Ki-67 (336 bp): 5'-AGC CAC CCT GCA GCA AGC AC-3' (forward primer); 5'-AGC CCA GCA GTG CTC CCA GT-3' (reverse primer). GAPDH (526 bp): 5'-AGG TCG GAG TCA ACG GAT TTG-3' (forward primer), 5'-GTG ATG GCA TGG ACT GTG GT-3' (reverse primer). GAPDH (230 bp): 5'-CTC TCT GCT CCT CCT GTT CGA CAG-3' (forward primer); 5'-GTG 


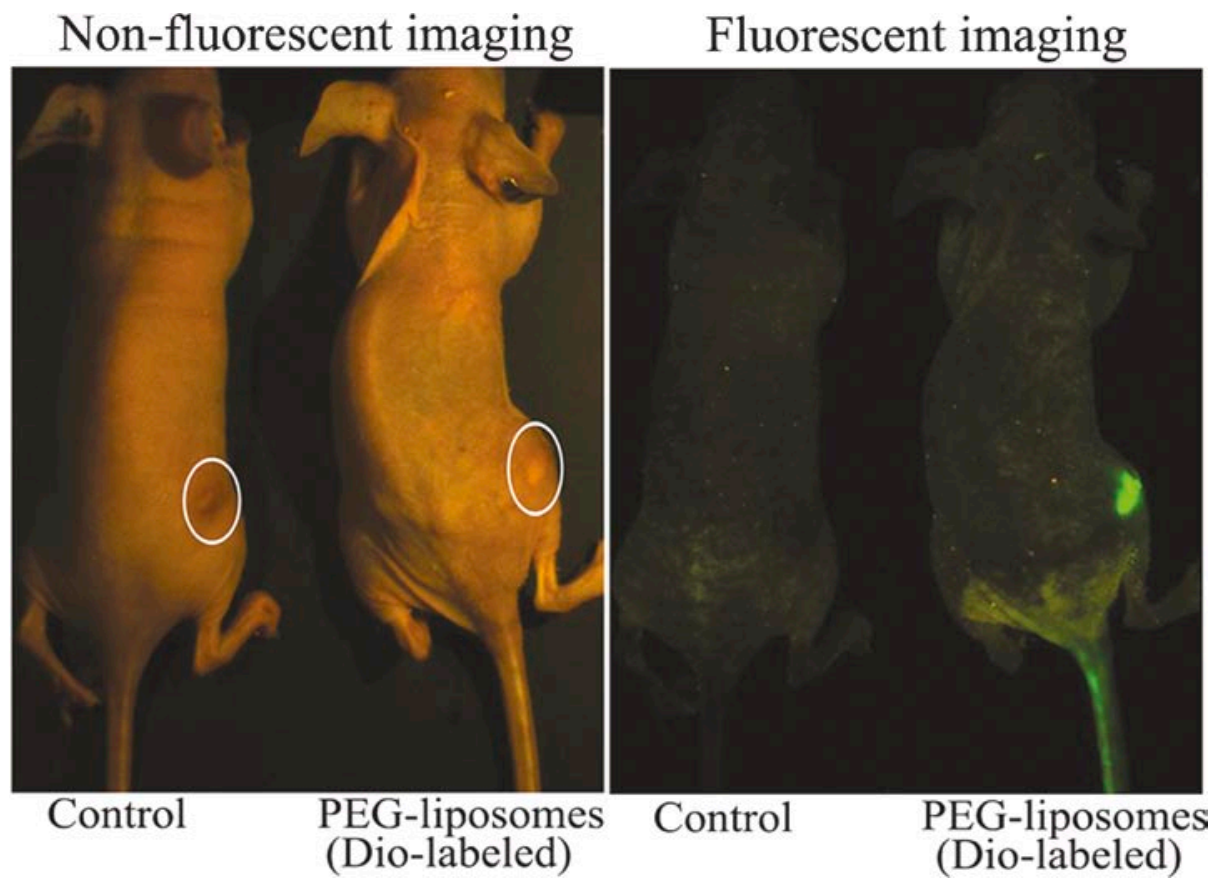

Figure 1. Dio-labeled PEG-liposomes accumulate in tumor tissue in vivo. On day 12 after tumor inoculation mice received an intravenous injection of fluorescently (Dio)-labeled empty PEG-liposomes (no drug) at a dose of $0.5 \mathrm{nmol}$ lipid/g. At 24 h post injection, in vivo imaging were taken, only intravenous injection of $5 \%$ dextrose solution as control.

GAA TCA TAT TGG AAC ATG T-3' (reverse primer). For the analysis of PCR products, $6 \mu$ of each PCR reaction was electrophoresed on $1.5 \%$ agarose gel containing $1 \%$ GoldView $^{\mathrm{TM}}$. Band intensity was analyzed with Image system (NIH, USA) and the GAPDH was used as an internal control to evaluate relative expression of Bcl-2, Bax, Caspase-3 and Ki-67.

Western blot analysis. For isolation of total protein extract, tumor tissues were washed with ice-cold PBS and lysed in RIPA lysis buffer (50 mM Tris with $\mathrm{pH} 7.4,150 \mathrm{mM} \mathrm{NaCl}, 1 \%$ Triton X-100, $1 \%$ sodium deoxycholate, $0.1 \%$ sodium dodecyl sulphate and $0.05 \mathrm{mM}$ EDTA) for $30 \mathrm{~min}$ on ice, and cell lysate was centrifuged $\left(12,000 \mathrm{x} \mathrm{g}\right.$, at $\left.4^{\circ} \mathrm{C}\right)$ for $10 \mathrm{~min}$. The supernatant was collected and protein content of extracted samples was measured using Bradford protein assay kit (BestBo-BeiBo, Beijing, China). All samples were stocked at $-80^{\circ} \mathrm{C}$ for further experiments.

Levels of target proteins including Bcl-2, Bax (1:200), P17 and $\beta$-actin (1:500) were determined by Western blot analysis using the respective antibodies stated above. Briefly, equal amount of proteins (50 $\mu \mathrm{g}$ /lane) was subjected to $15 \%$ SDS-polyacrylamide gel electrophoresis and transferred to polyvinylidene fluoride membranes (PVDF). The membranes were blocked with 5\% skim milk in PBS with $0.1 \%$ Tween-20 (PBST) for $1 \mathrm{~h}$, and incubated with primary antibodies overnight at $4^{\circ} \mathrm{C}$. Antibodies were detected by means of HRP-conjugated secondary antibody $(1: 2000)$ for $1 \mathrm{~h}$ at room temperature. Immunoreactive bands were visualized using Immobilon $^{\mathrm{TM}}$ Western chemiluminescent HRP substrate (Millipore, USA), and densitometric analysis was performed with the use of PDI Imageware System (Bio-Rad, Hercules, CA, USA).
Statistical analysis. The means and standard deviations were calculated, values are expressed as mean \pm SD. From the control and treated groups, the statistical comparisons were performed using SPSS 17.0 package (SPSS Inc., USA). One-way analysis of variance (ANOVA) and Student's t-test were carried out to determine statistical significance. Differences were considered significant at $\mathrm{p}<0.05$.

\section{Results}

Tumor tissue uptake Dio-labeled PEG-liposomes. Dio-labeled PEG-liposomes were intravenously injected via tail vein, then visualized in the tumor by an in vivo imaging system. After $24 \mathrm{~h}$ images were evaluated The fluorescence intensity distribution of tumor tissue in the animals is presented in green color fluorescence images (Fig. 1) indicating that PEG-liposomes accumulate in tumor tissue.

Intratumoral localization of the liposomes was determined in the tumor xenograft to evaluate the affinity of PEG-liposomes to angiogenic vessels in vivo. PEG-liposomes were observed around tumor angiogenic vessels, moreover tumor cells had taken up PEG-liposomes (Fig. 2).

Determination of L-oHP amount in solid tumors. The mouse tumor-xenografts were treated with intravenous injection of either free L-oHP or PEG-liposomal L-oHP to examine intratumoral L-oHP distribution (Fig. 3). Maximum intratumor accumulation of free L-oHP occurred at $2 \mathrm{~h}$ post-injection, it was $7.43 \pm 0.64 \mu \mathrm{g} / \mathrm{g}$, and gradually decreased to near zero levels by $48 \mathrm{~h}$ after injection. At $72 \mathrm{~h}$ free L-oHP was not detected. The value reached maximum at $24 \mathrm{~h}$ post-injection, on the contrary, with PEG-liposomal L-oHP, it was $9.37 \pm 0.79 \mu \mathrm{g} / \mathrm{g}$, 


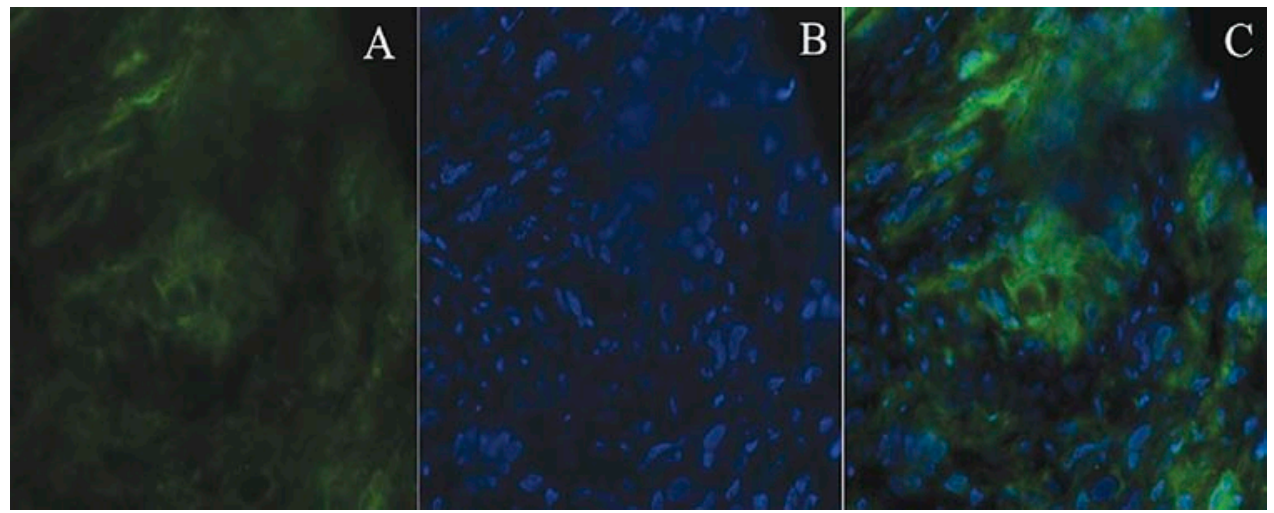

Figure 2. Intratumoral localization of the PEG-liposomes. The mice were intravenously injected with Dio-labeled liposomes, at $24 \mathrm{~h}$ after injection, the tumors were dissected, and then frozen-sections $(10 \mu \mathrm{m})$ were prepared. Immunofluorescence staining for Hochest ${ }_{33324}$ was performed to visualize the nucleus. Green images show liposomal distribution (A), and blue images indicate nucleus regions (B), panel C represents the merged images (Hochest ${ }_{33324}, \mathrm{x}_{400)}$

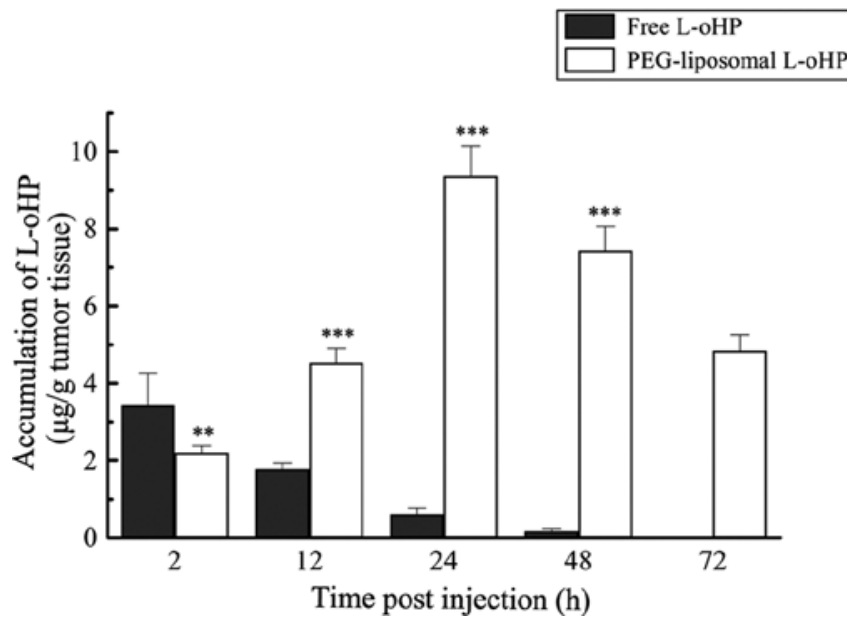

Figure 3. In vivo tumor accumulation of L-oHP. On day 12 after tumor inoculation, mice received an intravenous injection of free L-oHP $(5 \mu \mathrm{g} / \mathrm{g})$, PEG-liposomal L-oHP (containing $5 \mu \mathrm{g} / \mathrm{g} \mathrm{L}$-oHP), respectively. At 2, 12, 24, 48 or $72 \mathrm{~h}$ post injection, tumors were excised. The amount of L-oHP accumulated in tumor tissues was measured by HPLC system. Data represent the mean $\pm \mathrm{SD}(\mathrm{n}=3) .{ }^{* *} \mathrm{p}<0.01,{ }^{* * *} \mathrm{p}<0.001$ against free L-oHP. moreover, at 48 and $72 \mathrm{~h}$ the level was still higher than the maximal level obtained with free L-oHP.

Assessment tumor volume and survival rate. Rapid tumor growth was observed in mice in the control group (Fig. 4), however, signficant tumor growth suppression was observed in mice treated with PEG-liposomal L- oHP (Fig. 5A). The PEG-liposomal L-oHP showed the strongest effect on the survival time, all the mice treated with PEG-liposomal L-oHP became long-term survivors (Fig. 5B). In addition, noticeable cachexia condition was observed in control group at 30 days. Through the therapeutic experiment, bodyweight loss was not observed in any of the treated groups, but was significant in the controls (data not shown). These results suggest that treatment with PEG-liposomal L-oHP improves the median survival time (MST) of tumor-bearing mice without causing remarkable toxicity.

Apoptosis and expression of $\mathrm{Ki}-67$ in tumor tissue. Apoptotic body index (ABI) was assessed by a standard TUNEL method. Proliferating activity of a neoplasm can be assessed
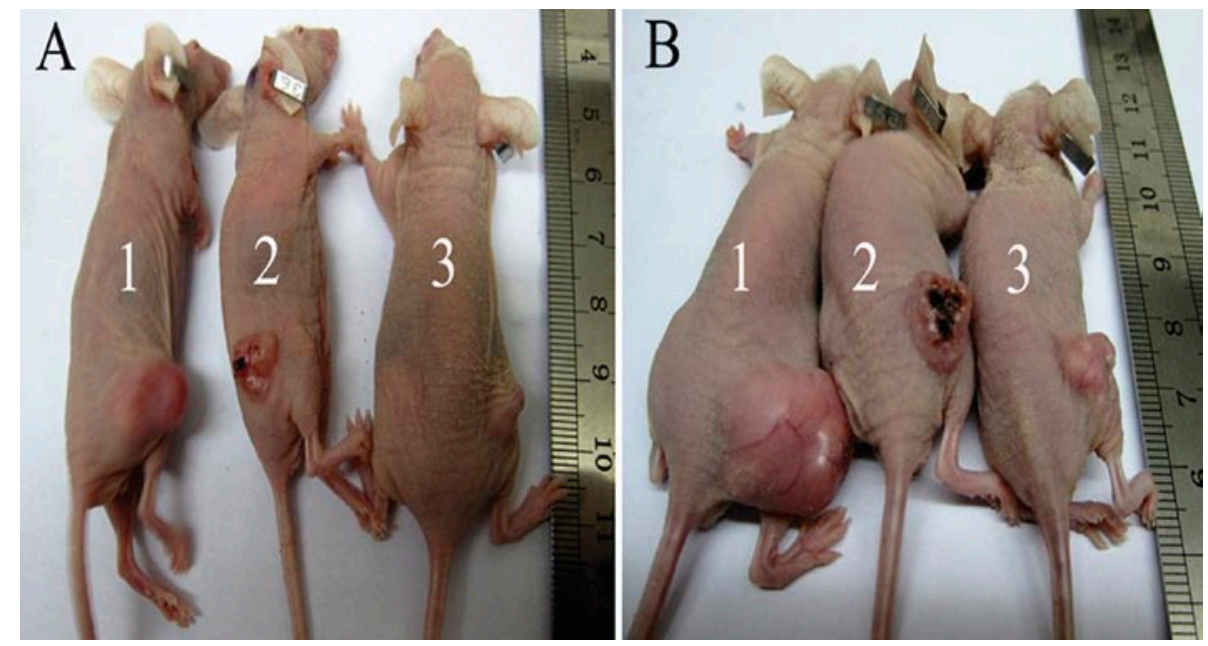

Figure 4. Tumor growth observation after treatment. On day 14 after tumor inoculation, either free L-oHP (2), PEG-liposomal L-oHP (3), or 5\% dextrose solution (control) (1) was administered via tail vein at an L-oHP dose of $5 \mu \mathrm{g} / \mathrm{g}$. The tumor growth was observed. (A) 1 week after treatment. (B) Three weeks after treatment. 

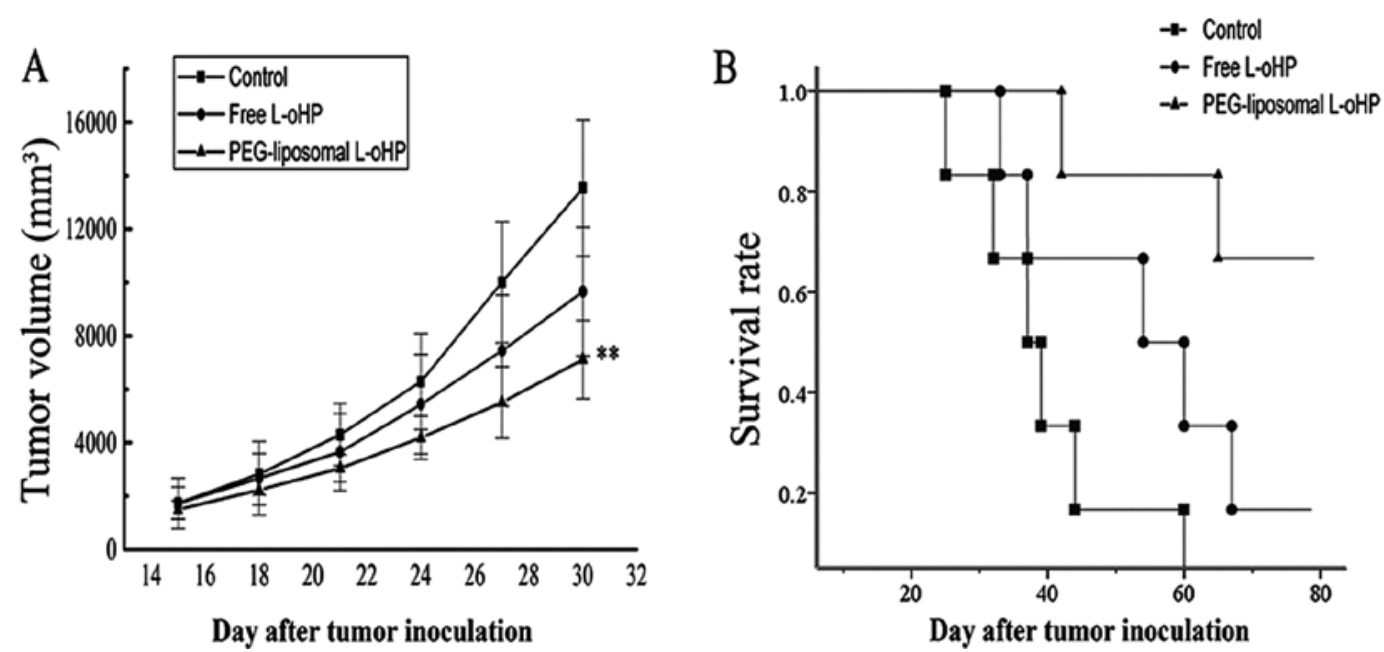

Figure 5. Tumor growth suppression and survival time. Antitumor activity as assessed by tumor size and survival of tumor-bearing mice. (A) Tumor volume, data represent the mean $\pm \mathrm{SD}(\mathrm{n}=6){ }^{* *} \mathrm{p}<0.01$ compared with the other group. (B) Survival of tumor-bearing mice.

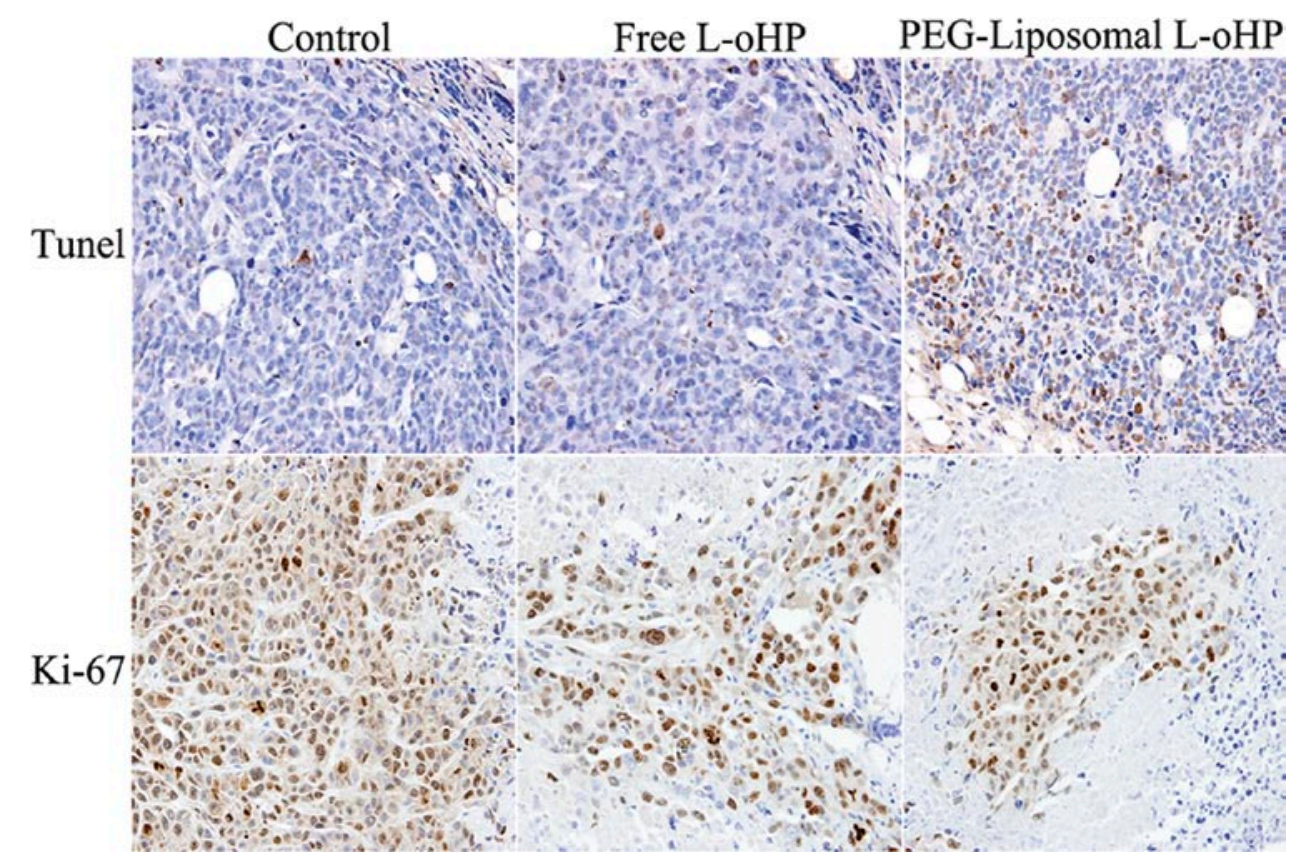

Figure 6. Apoptosis and proliferation expression. Photomicrographs showing apoptotic bodies [TUNEL(+) cells], and Ki-67 expression. In Ki-67 (+) cells karyokinesis was observed (DAB, $\mathrm{x} 400)$.

by measuring the tumor growth fraction, by immunohistochemically identifying specific cell cycle-related antigens.

In this study, the cellular proliferation was suppressed, in contrast, the apoptosis increased with accumulation of drug, and amount of ABI were observed in the tumors (Fig. 6), ABI values were $5.83 \pm 1.13 \%, 14.88 \pm 3.25 \%, 35.94 \pm 5.04 \%$. There was a strong correlation of increased $\mathrm{ABI}$ values when treated with PEG-liposomal L-oHP $(p<0.01)$. We further evaluated the cell proliferation rate using $\mathrm{Ki}-67$ antibody. On the whole, the proliferation rates were low after treatment with PEG-liposomal L-oHP. The proliferation index was $28.74 \pm 5.77 \%, 12.16 \pm 3.93 \%, 7.56 \pm 1.62 \%$, respectively $(\mathrm{p}<0.01)$.
Bcl-2, Bax and Caspase-3 mRNA and protein expression in tumor tissue. Bcl-2, Bax and Caspase-3 are association with apoptosis, Ki-67 as a proliferation marker. To elucidate whether the growth inhibitory effect of PEG-liposomal L-oHP was attributable to the induction of apoptosis, Bcl-2, Bax and Caspase-3 were analyzed by RT-PCR or Western blotting in tumor tissue. Our experiments show that mRNA expression levels of $\mathrm{Bcl}-2$ and $\mathrm{Ki}-67$ was remarkably decreased in PEG-liposomal L-oHP group, compared with free L-oHP, whereas, Bax and Caspase-3 mRNA were increased (Fig. 7A). Protein expression tendency of Bcl-2, Bax and P17 were coincidented with mRNA expression (Fig. 7B). These result indicated that apoptosis was strongly induced by PEG-liposomal L-oHP. 


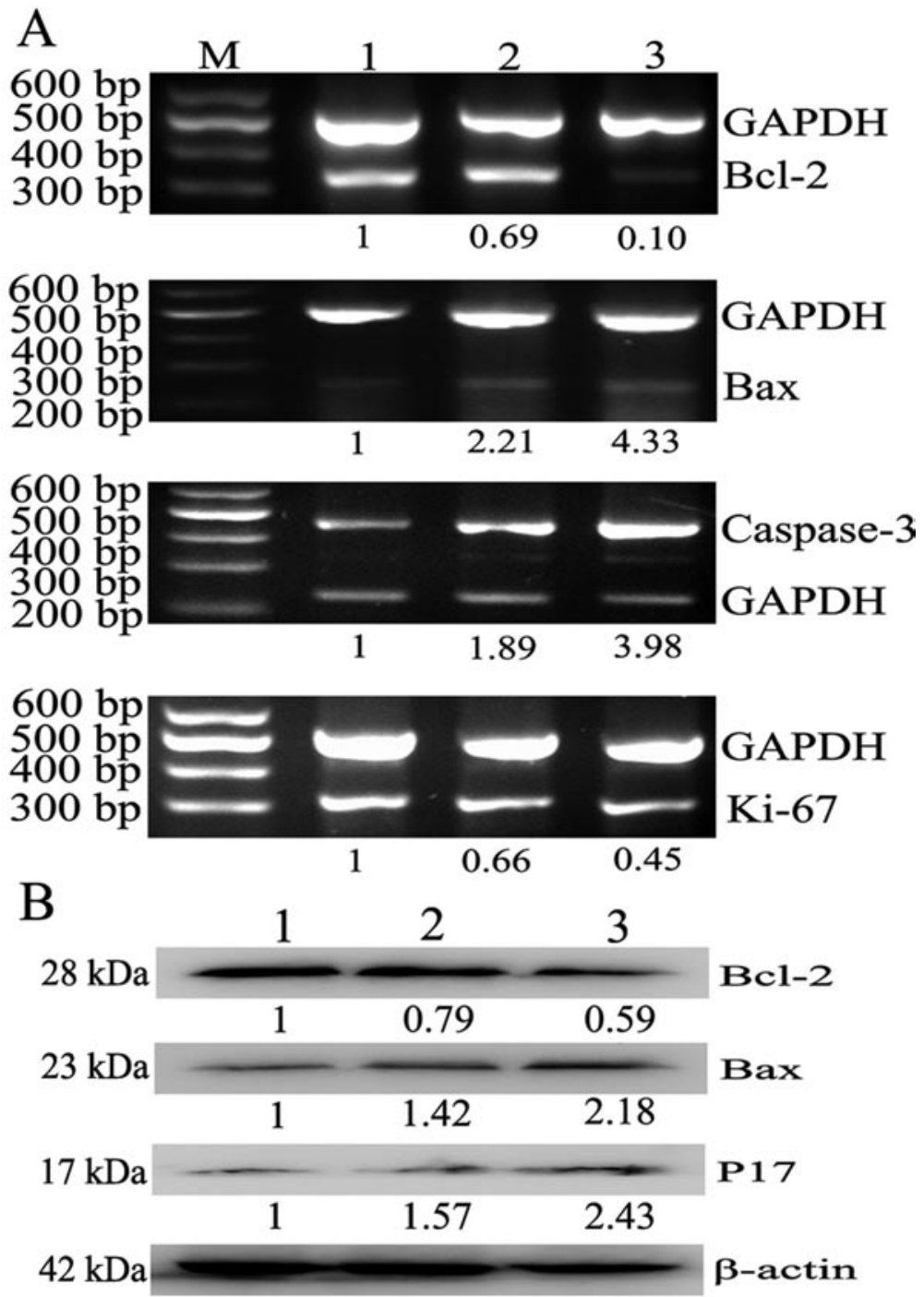

Figure 7. The mRNA, protein expression of Bcl-2, Bax, Caspase-3 and Ki-67 in tumor tissue. After treatment with free L-oHP (2), PEG-liposomal L-oHP (3), or 5\% dextrose solution (1), the total mRNA and protein was extracted from tumor tissue. M, DNA marker. (A) The mRNA expression. (B) The protien expression. Data are expressed as the mean $\pm \mathrm{SD}(\mathrm{n}=3) .{ }^{* * *} \mathrm{p}<0.01$ compared with the other group.

\section{Discussion}

In this study, we evaluated antitumor effectiveness of PEG-liposomal L-oHP in in vivo experiments. PEG modification of liposomes, as carriers of chemotherapeutic agents, can change the distribution of these agents within the body and decrease their toxicity.

The non-selectivity of cytotoxic drugs between normal tissue and the pathological site poses a challenge for the treatment strategy of tumors. To obtain increased therapeutic efficacy for tumors, a drug carrier must achieve increased delivery of the drug in the tumor tissue, in other words, it must enhance interaction of the drug and the subsequent internalization by tumor cells. Therefore, drug-loaded liposomes offer new approaches for the treatment of colorectal cancer.

In prophase stage, we found that tumor cells could take up large numbers of PEG-liposomes, as reported by Abu et al (14). Further research demonstrated that PEG-modified liposomes are mostly through the vesicular organelles, then preferentially taken up by angiogenic tumor endothelium (15). Through in vivo imaging system, our experiments showed that PEG-liposomes efficiently accumulated in the tumor tissue. This result coincidence with Abu et al (14). After treatment with PEG-liposomal L-oHP, accumulation of L-oHP was persistent, reached higher level from 24 to $72 \mathrm{~h}$. However, with L-oHP treatment it rapidly decreased. Thus suggesting that PEG-liposome-containing agent can assemble and slowly release drugs in tumor tissue. The tumor growth suppression was significant, and showed stronger effect on the survival time than free oxaliplatin.

To further demonstrate the treatment availability of PEG-liposomal L-oHP, apoptosis and proliferation were detected. Apoptosis, (programmed cell death), is characterized by cytoplasmic fragmentation and nuclear condensation, and contributes to both physiological and pathological processes (16-19). It was assessed by a standard TUNEL method. Proliferating activity of a neoplasm can be assessed by measuring the tumor growth fraction, a widely used marker for proliferating cells is the $\mathrm{Ki}-67$ antigen, which is expressed 
in nuclei during all phases of the cell cycle except G0, and its presence has been related to tumor recurrence, stage, and grade $(20,21)$. The proportion of Ki-67-labeled cells in a given cell population (Ki-67 index) provides a measure of the growth fraction (22). We found apoptosis of tumor cells in tumor-bearing nude mice inducing high $\mathrm{ABI}$ when treated with PEG-liposomal L-oHP. In the tumors ABI values varied from 5 to $60 \%$ (23), our data showed from 5 to $40 \%$. However, expression of Ki-67 was lower, and region of necrosis with less $\mathrm{Ki}-67$ antigent.

The apoptosis and proliferation in the current study, a consistent with other studies $(23,24)$, suggesting that apoptosis might reflect not only cell loss but also a proliferative activity. In addition, various proteins that operate in the cell cycle checkpoints are also regulators of apoptosis $(25,26)$. To further evaluate the antitumor effectiveness of PEG-liposomal L-oHP, Bcl-2, Bax and Caspase-3 were evaluated. Bcl-2, Bax are associated with apoptosis, necrosis, and autophagy, and regulate all major types of cell death (27-29). Caspase-3 is the final executor of apoptosis $(30,31)$. After treatment with PEG-liposomal L-oHP, apoptosis of tumor cells in tumor-bearing nude mice was induced. Expression of Bcl-2 was down-regulated, and, Bax and Caspase-3 significantly up-regulated. Expression of protein coincide with mRNA, the results of the present study suggested that protein or mRNA expression correlated with apoptosis or proliferation as described above.

The liposomal L-oHP formulation has a potent in vivo antitumor activity, presumably via a dual targeting approach against both tumor endothelial cells and tumor cells $(32,33)$. The PEG-liposomal L-oHP accumulated in the tumor tissue, is most likely via mechanism of the increased amount of time that the liposomes circulate in the blood and the leaky nature of the microvasculature in solid tumor tissues. Following their uptake by endothelial cells as well as tumor cells, liposomes are degraded, while intracellular drug delivery increases the concentration of drug within cells and slows drug efflux (34-36). This study indicates that liposome encapsulation of chemotherapeutic drugs enhances their damaging effects on tumor cells. It may have strong potential to overcome some major limitations in conventional anticancer chemotherapy, and maybe provide a rationale for clinical application of CRC.

\section{Acknowledgements}

This study was supported by a grant (no. 09-2-12) from the Health Administration of Chongqing. We thank Xin-Hui Jiang for expert technical assistance of HLPC, we are grateful to the Central Lab, the Ophthalmological Lab of Chongqing Medical University First Affiliated Hospital and the College of Life Science of Chongqing Medical University for their technical supports.

\section{References}

1. Nobili S, Checcacci D, Filippelli F, et al: Bimonthly chemotherapy with oxaliplatin, irinotecan, infusional 5-fluorouracil/ folinic acid in patients with metastatic colorectal cancer pretreated with irinotecan-or oxaliplatin-based chemotherapy. J Chemother 20: 622-631, 2008.

2. Des GG, Lecaille C, Mariani P, et al: Prognostic impact of microsatellite instability in colorectal cancer patients treated with adjuvant FOLFOX. Anticancer Res 30: 4297-4301, 2010.
3. Tampellini M: Pharmacoeconomic aspects of FOLFIRI or FOLFOX regimens administered with a fully ambulatory pump compared to the day hospital setting. Tumori 96: 438-442, 2010.

4. Pasetto LM, D'Andrea MR, Rossi E, et al: Oxaliplatin-related neurotoxicity: how and why? Crit Rev Oncol Hematol 59: 159-168, 2006.

5. Michor F, Iwasa Y, Lengauer C, et al: Dynamics of colorectal cancer. Semin Cancer Biol 15: 484-493, 2005.

6. Pietrangeli A, Leandri M, Terzoli E, et al: Persistence of high-dose oxaliplatin-induced neuropathy at long-term followup. Eur Neurol 56: 13-16, 2006.

7. Hussain S, Pluckthun A, Allen TM, et al: Antitumor activity of an epithelial cell adhesion molecule targeted nanovesicular drug delivery system. Mol Cancer Ther 6: 3019-3027, 2007.

8. Sun W, Zou W, Huang G, et al: Pharmacokinetics and targeting property of TFu-loaded liposomes with different sizes after intravenous and oral administration. J Drug Target 16: 357-365, 2008.

9. Allen TM, Hansen C, Martin F, et al: Liposomes containing synthetic lipid derivatives of poly(ethylene glycol) show prolonged circulation half-lives in vivo. Biochim Biophys Acta 1066: 29-36, 1991.

10. Klibanov AL, Maruyama K, Beckerleg AM, et al: Activity of amphipathic poly(ethylene glycol) 5000 to prolong the circulation time of liposomes depends on the liposome size and is unfavorable for immunoliposome binding to target. Biochim Biophys Acta 1062: 142-148, 1991.

11. Allen C, Dos Santos N, Gallagher R, et al: Controlling the physical behavior and biological performance of liposome formulations through use of surface grafted poly(ethylene glycol). Biosci Rep 22: 225-250, 2002.

12. Matsumura Y and Maeda $\mathrm{H}$ : A new concept for macromolecular therapeutics in cancer chemotherapy: mechanism of tumoritropic accumulation of proteins and the antitumor agent Smancs. Cancer Res 46: 6387-6392, 1986.

13. Yang C, Liu HZ and Fu ZX: Effect on apoptosis of human colorectal cancer SW480 cells induced by oxaliplatin longcirculating liposomes. J Third Mil Med Uni 33: 368-371, 2011.

14. Abu Lila AS, Kizuki S, Doi Y, et al: Oxaliplatin encapsulated in PEG-coated cationic liposomes induces significant tumor growth suppression via a dual-targeting approach in a murine solid tumor model. J Control Release 137: 8-14, 2009.

15. Thurston G, Mclean JW, Rizen M, et al: Cationic liposomes target angiogenic endothelial cells in tumours and chronic inflammation in mice. J Clin Invest 101: 1401-1413, 1998.

16. Bosman FT, Visser BC and van Oeveren J: Apoptosis: pathophysiology of programmed cell death. Pathol Res Pract 192: 676-683, 1996.

17. Cummings MC, Winterford CM and Walker NI: Apoptosis. Am J Surg Pathol 21: 88-101, 1997.

18. Staunton MJ and Gaffney EF: Apoptosis. Basic concepts and potential significance in human cancer. Arch Pathol Lab Med 122: 310-319, 1998.

19. Geske FJ and Gerschenson LE: The biology of apoptosis. Hum Pathol 32: 1029-1038, 2001.

20. Scott RJ, Hall PA, Haldane JS, et al: A comparison of immunohistochemical markers of cell proliferation with experimentally determined growth fraction. J Pathol 165: 173-178, 1991.

21. Kruger S and Muller H: Correlation of morphometry, nucleolar organizer regions, proliferating cell nuclear antigen and Ki-67 antigen expression with grading and staging in urinary bladder carcinomas. Br J Urol 75: 480-484, 1995.

22. Reed JC: Bcl-2-family proteins and hematologic malignancies: history and future prospects. Blood 111: 3322-3330, 2008.

23. Tsamandas AC, Kardamakis D, Tsiamalos P, et al: The potential role of Bcl-2 expression, apoptosis and cell proliferation (Ki-67 expression) in cases of gastric carcinoma and correlation with classic prognostic factors and patient outcome. Anticancer Res 29: 703-709, 2009.

24. Scopa CD, Tsamandas AC, Zolota V, et al: Potential role of bcl-2 and $\mathrm{Ki}-67$ expression and apoptosis in colorectal carcinoma $\mathrm{A}$ clinicopathologic study. Dig Dis Sci 48: 1990-1997, 2003.

25. Soini Y, Paakko P and Letho V-P: Histopathological evaluation of apoptosis in cancer. Am J Pathol 153: 1041-1053, 1998.

26. Tabete S, Ishida M, Kasagi N, et al: Apoptosis occurs more frequent in metastatic foci than in primary lesions of human colorectal carcinomas: analysis by terminaldeoxynucleotidyltransferase-mediated dUTP-biotin nick end labeling. Int J Cancer 65: 173-177, 1996.

27. Chipuk JE, Fisher JC, Dillon CP, et al: Mechanism of apoptosis induction by inhibition of the anti-apoptotic BCL-2 proteins. Proc Natl Acad Sci USA 105: 20327-20332, 2008. 
28. Luo X, Budihardjo I, Zou H, et al: Bid, a Bcl-2 interacting protein, mediates cytochrome c release from mitochondria in response to activation of cell surface death receptors Cell 94: 481-490, 1998.

29. Adams JM and Cory S: The Bcl-2 protein family: arbiters of cell survival. Science 281: 1322-1326, 1998.

30. Jaattela $\mathrm{M}$ and Tschopp J: Caspase-independent cell death in T lymphocytes. Nat Immunol 4: 416-423, 2003.

31. Walters J, Pop C, Scott FL, et al: A constitutively active and uninhibitable caspase-3 zymogen efficiently induces apoptosis. Biochem J 424: 335-345, 2009.

32. Lee CM, Tanaka T, Murai T, et al: Novel chondroitin sulfatebinding cationic liposomes loaded with cisplatin efficiently suppress the local growth and liver metastasis of tumor cells in vivo. Cancer Res 62: 4282-4288, 2002.
33. Jain A, Jain SK, Ganesh N, et al: Design and development of ligand-appended polysaccharidic nanoparticles for the delivery of oxaliplatin in colorectal cancer. Nanomedicine 6: 179-190, 2010.

34. Saito R, Bringas JR, McKnight TR, et al: Distribution of liposomes into brain and rat brain tumor models by convection-enhanced delivery monitored with magnetic resonance imaging. Cancer Res 64: 2572-2579, 2004

35. Lappalainen K, Miettinen R, Kellokoski J, et al: Intracellular distribution of oligonucleotides delivered by cationic liposomes: light and electron microscopic study. J Histochem Cytochem 45: 265-274, 1997.

36. Atobe K, Ishida T, Ishida E, et al: In vitro efficacy of a sterically stabilized immunoliposomes targeted to membrane type 1 matrix metalloproteinase (MT1-MMP). Biol Pharm Bull 30: 972-978, 2007. 